\title{
Uninterrupted monitoring of drug effects in human-induced pluripotent stem cell-derived cardiomyocytes with bioluminescence $\mathrm{Ca}^{2+}$ microscopy
}

Kazushi Suzuki ${ }^{1 \dagger}$, Takahito Onishi ${ }^{1 \dagger}$, Chieko Nakada², Shunsuke Takei², Matthew J. Daniels ${ }^{3}$, Masahiro Nakano ${ }^{1}$, Tomoki Matsuda ${ }^{1}$ and Takeharu Nagai ${ }^{{ }^{*}}$

\begin{abstract}
Objective: Cardiomyocytes derived from human-induced pluripotent stem cells are a powerful platform for highthroughput drug screening in vitro. However, current modalities for drug testing, such as electrophysiology and fluorescence imaging have inherent drawbacks. To circumvent these problems, we report the development of a bioluminescent $\mathrm{Ca}^{2+}$ indicator $\mathrm{GmNL}\left(\mathrm{Ca}^{2+}\right)$, and its application in a customized microscope for high-throughput drug screening.

Results: $\mathrm{GmNL}\left(\mathrm{Ca}^{2+}\right)$ gives a 140\% signal change with $\mathrm{Ca}^{2+}$, and can image drug-induced changes of $\mathrm{Ca}^{2+}$ dynamics in cultured cells. Since bioluminescence requires application of a chemical substrate, which is consumed over $\sim 30$ min we made a dedicated microscope with automated drug dispensing inside a light-tight box, to control drug addition. To overcome thermal instability of the luminescent substrate, or small molecule, dual climate control enables distinct temperature settings in the drug reservoir and the biological sample. By combining $\mathrm{GmNL}\left(\mathrm{Ca}^{2+}\right)$ with this adaptation, we could image spontaneous $\mathrm{Ca}^{2+}$ transients in cultured cardiomyocytes and phenotype their response to well-known drugs without accessing the sample directly. In addition, the bioluminescent strategy demonstrates minimal perturbation of contractile parameters and long-term observation attributable to lack of phototoxicity and photobleaching. Overall, bioluminescence may enable more accurate drug screening in a high-throughput manner.
\end{abstract}

Keywords: hiPSC, Cardiomyocytes, Drug screening, Bioluminescence, $\mathrm{Ca}^{2+}$, Microscope

\section{Introduction}

For decades, a major bottleneck in drug development has been limited availability of patient-derived tissues or cells. This changed with the establishment of humaninduced pluripotent stem cells (hiPSC) [1,2], which can now be differentiated into virtually all cell types in vitro. Patient derived disease-specific hiPSCs recapitulate many disease phenotypes in culture, which may therefore

\footnotetext{
*Correspondence: ng1@sanken.osaka-u.ac.jp

${ }^{\dagger}$ Kazushi Suzuki and Takahito Onishi Contributed equally to this work

${ }^{1}$ The Institute of Scientific and Industrial Research, Osaka University, 8-1 Mihogaoka, Ibaraki, Osaka 567-0047, Japan

Full list of author information is available at the end of the article
}

serve as a valuable platform for drug discovery or toxicology testing [3]. This is especially true for the hiPSCderived cardiomyocytes (hiPSC-CMs) which may enable a paradigm shift in toxicity testing [4]. However thus far they have failed to completely recapitulate established real-world patient based toxicology results in contemporary platforms $[5,6]$.

Drug-induced changes in hiPSC-CMs can be detected by many methods including classical and automated electrophysiology or established fluorescence imaging modalities [7, 8]. However, the inherent low-throughput of electrophysiological techniques and physiological perturbation arising from phototoxicity 
of fluorescence excitation may introduce limitations such as recording duration (precluding chronic toxicity studies), or artefacts.

By contrast biocompatibility of bioluminescence emission is robustly demonstrated in many eukaryotic phyla, and as such may provide a better short or long term imaging solution. Bioluminescent proteins (BPs) generate detectable emissions by catalyzing a chemical reaction which consumes a bioluminescent substrate while releasing a photon in the process. This makes them totally independent of external light, reducing phototoxicity. Recent developments of bright BPs such as Nano-lantern, a chimera of Renilla luciferase (Rluc) variant and a fluorescent protein (FP), enable bioluminescence imaging with high signal-to-noise ratio comparable to fluorescence imaging $[9,10]$. However, performing bioluminescence imaging at scale is still limited. The faint light associated with bioluminescence requires placement inside an opaque box in order to exclude background light. This factor makes it difficult to test the enormous chemical libraries in a high-throughput manner.

Here we develop a new bioluminescent $\mathrm{Ca}^{2+}$ indicator $\mathrm{GmNL}\left(\mathrm{Ca}^{2+}\right)$ for hiPSC-CM imaging together with a customized light-tight box which contains a liquid dispenser and regional temperature control and can easily be installed on existing fluorescence microscopy. Combining $\mathrm{GmNL}\left(\mathrm{Ca}^{2+}\right)$ with the environmental modifications we demonstrate prolonged visualization of $\mathrm{Ca}^{2+}$ transients is improved by bioluminescence compared to fluorescence imaging. Consequently this strategy in hiPSC-CMs may bring value to drug development, particularly in chronic toxicology studies where there is an unmet need.

\section{Main text}

\section{Materials and methods}

Gene construction of $\mathrm{GmNL}\left(\mathrm{Ca}^{2+}\right)$

General molecular biology experiments were conducted as described [11]. The sequences of all the oligonucleotides (Hokkaido System Science, Hokkaido, Japan) used in this study are provided in Additional file 1. Each of the cDNAs of C-terminally deleted Gamillus [12] mutants $(\triangle \mathrm{C} 8-11)$ were amplified by PCR and digested with BamHI and KpnI. The digested PCR fragments were cloned in-frame into the KpnI/EcoRI sites of Nano-lantern $\left(\mathrm{Ca}^{2+}\right) \_600 /$ pRSETB (Addgene\#51976) for bacterial expression. To express the $\mathrm{GmNL}\left(\mathrm{Ca}^{2+}\right)$ in mammalian cells, PCR-amplified $\operatorname{GmNL}\left(\mathrm{Ca}^{2+}\right)$ genes were inserted into a pcDNA3 mammalian expression vector using BamHI and EcoRI sites.

\section{Protein purification and characterization}

Recombinant Gamillus-based $\mathrm{NL}\left(\mathrm{Ca}^{2+}\right)$ _variant proteins with $\mathrm{N}$-terminal polyhistidine tags were expressed in $E$. coli. (JM109(DE3)), purified, and spectroscopically characterized as described [11].

\section{Adeno-associated virus (AAV) production}

For the Adeno associated virus expression system, pHelper and pAAV-DJ were obtained from Cell Biolabs, Inc. (San Diego, CA, USA). The cDNA of $\mathrm{GmNL}\left(\mathrm{Ca}^{2+}\right)$ was replaced with ArchT-GFP sequence in pAAV-CAGArchT-GFP (Addgene\#29777). AAV production followed the manufacturer's protocol. The titer of AAV vector was determined by fluorescence titration assay. Briefly, HEK293T cells were transduced with a range of dilutions of AAV encoding $\mathrm{GmNL}\left(\mathrm{Ca}^{2+}\right) .48 \mathrm{~h}$ post transduction, the percentage of infected cells were assayed with fluorescence of Gamillus moiety inside $\mathrm{GmNL}\left(\mathrm{Ca}^{2+}\right)$ using fluorescence microscopy. To ensure a single infection event per cell, the dilution with less than $40 \%$ fluorescent-positive cells was adopted for calculation of titer. The virus titer was approximately $1 \times 10^{8}$ Infectious units (IFU)/ml.

\section{Customized bioluminescence microscopy}

A bespoke assay environment for bioluminescence imaging was developed for a Ti-E microscope (Nikon Corporation, Tokyo, Japan) equipped with a x10PlanFluor (NA 0.3) objective lens and a stage modified for onstage environmental control (Tokai Hit., Co, Ltd., Shizuoka, Japan). Drug addition and medium exchange was carried out using PROcellcare 5030 and PPPump 2010 MiMEDA enclosed in light-tight box manufactured for this study (Tokai Hit., Co, Ltd.). Fluorescence and bioluminescence images were acquired with an EMCCD camera iXon-3 (Andor Technology, Belfast, Northern Ireland) using the image acquisition software NIS-Elements 4.60 (Nikon Corporation).

\section{hiPSC-CM culture and imaging}

iCell $^{\circledR}$ Cardiomyocytes (Cellular Dynamics International, Madison, WI, USA) were purchased, and aggregates of hiPSC-CMs were prepared as spheroids. hiPSC-CMs were treated with $10 \mu \mathrm{l}$ of crude AAV solution $\left(1 \times 10^{6}\right.$ IFU per aggregates of hiPSC-CMs $)$ for 1-5 weeks before observation. The cells were incubated at $37{ }^{\circ} \mathrm{C}, 5 \% \mathrm{CO}_{2}$ and culture medium replaced every 3 days. Just before observation hiPSC-CMs were washed with Tyrodes solution (Sigma-Aldrich, St. Louis, MO, USA) and exchanged for $20 \mu \mathrm{M}$ coelenterazine-h (Wako Pure Chemical Industries, Osaka, Japan) containing Tyrode solution. For $\mathrm{Ca}^{2+}$ imaging using Fluo4, 5.0 $\mu \mathrm{M}$ 
Fluo4-AM (Thermo Fisher Scientific, Waltham, MA, USA) was loaded onto hiPSC-CMs in Tyrode solution supplemented with $1 x$ PowerLoad (provided with Fluo4AM) for $1 \mathrm{~h}$ at $37^{\circ} \mathrm{C}$. Fluo 4 imaging was conducted with an FESH0700 IR cut-off filter (Thorlabs, Newton, NJ, USA) and LED505-C-FL (Nikon Corporation) including Ex500/20 excitation filter, DM515 dichroic mirror and EM535/30 emission filter. The images of $\mathrm{GmNL}\left(\mathrm{Ca}^{2+}\right)$ were acquired with 50 or $30 \mathrm{~ms}$ exposure times for comparison with Fluo4 or drug screening, respectively. For bioluminescence, $8 \times 8$ binning was applied to increase photon counts for each pixel.

For drug studies, the basal $\mathrm{Ca}^{2+}$ transients of hiPSC$\mathrm{CM}$ were recorded, followed by drug incubation for $10 \mathrm{~min}$, and repeated recording of the $\mathrm{Ca}^{2+}$ transient. To preserve drug activity, they were initially kept at $4{ }^{\circ} \mathrm{C}$ on one side of the chamber and just before use, the temperature was quickly raised to $37^{\circ} \mathrm{C}$ to prevent thermal drift. The information of all the drugs used in this study are provided in Additional file 2.

\section{Results}

\section{Development and characterization of a bioluminescent $\mathrm{Ca}^{2+}$ indicator}

In Nano-lantern, the excited energy produced by an Rluc variant is efficiently transferred to the adjacent FP by FRET. Since the FP has a higher quantum yield (QY) the emitted photon number increases. It was possible to introduce a calcium sensor domain into Nano-lantern to form Nano-lantern $\left(\mathrm{Ca}^{2+}\right)$, but this reduced brightness significantly [9]. To restore brightness we explored fusions to the recently characterized green FP Gamillus [12] which has the highest QY among reported GFPs. We first swapped Venus from Nano-lantern $\left(\mathrm{Ca}^{2+}\right)$ with various C-terminal truncated Gamillus $(\Delta 8-11)$ constructs to improve FRET efficiency. The resultant fusion proteins are designated as Gamillus-based $\mathrm{NL}\left(\mathrm{Ca}^{2+}\right)$ _variants hereafter (Fig. 1a). Of the tested Gamillus-based $\mathrm{NL}\left(\mathrm{Ca}^{2+}\right)$ vvariants, we found that the $\Delta 9$ deletion mutant exhibited a $140 \%$ signal change with comparable brightness to $\mathrm{YNL}\left(\mathrm{Ca}^{2+}\right)$ when $\mathrm{Ca}^{2+}$-bound (Fig. 1b). $\mathrm{Ca}^{2+}$ titration revealed that the dissociation constant $\left(K_{\mathrm{d}}\right)$ for $\mathrm{Ca}^{2+}$ was $240 \mathrm{nM}$. To compare the performance of these $\mathrm{Ca}^{2+}$ indicators to a known standard, we expressed each Gamillus-based $\mathrm{NL}\left(\mathrm{Ca}^{2+}\right)$ _variant in HeLa cells. Upon stimulation with histamine, an acute $\mathrm{Ca}^{2+}$ spike followed by $\mathrm{Ca}^{2+}$ oscillations with smaller amplitudes were detected with sampling rates up to $10 \mathrm{~Hz}$ (Additional file 3).

\section{Customized bioluminescence microscopy}

Figure 2a shows an inverted microscope customized for drug screening using bioluminescence imaging. The system is made of a stage-top incubator, and an automatic dispenser inside a light-tight box, enabling drug preparation, drug addition, and medium exchange (Fig. 2b). This

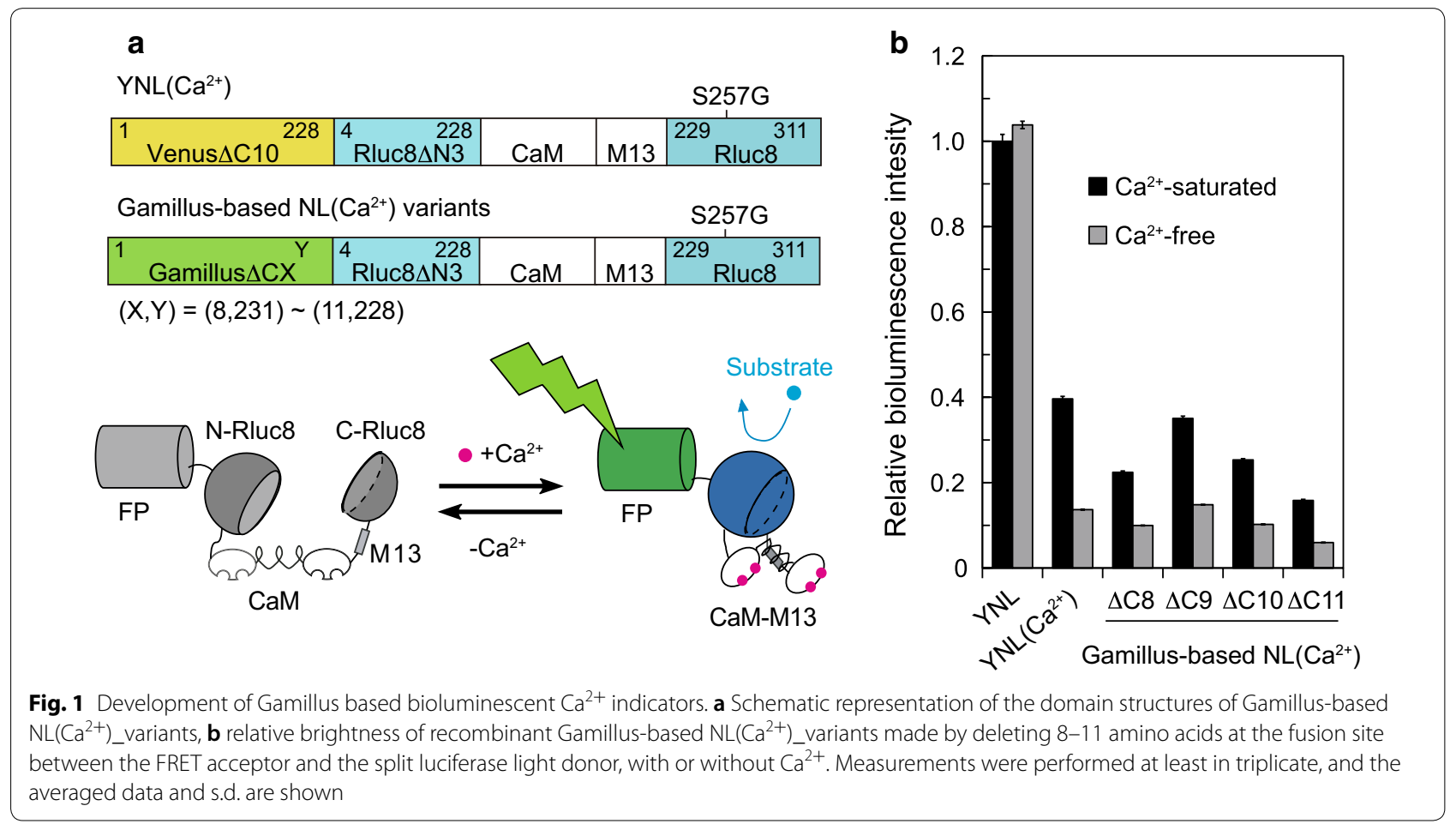



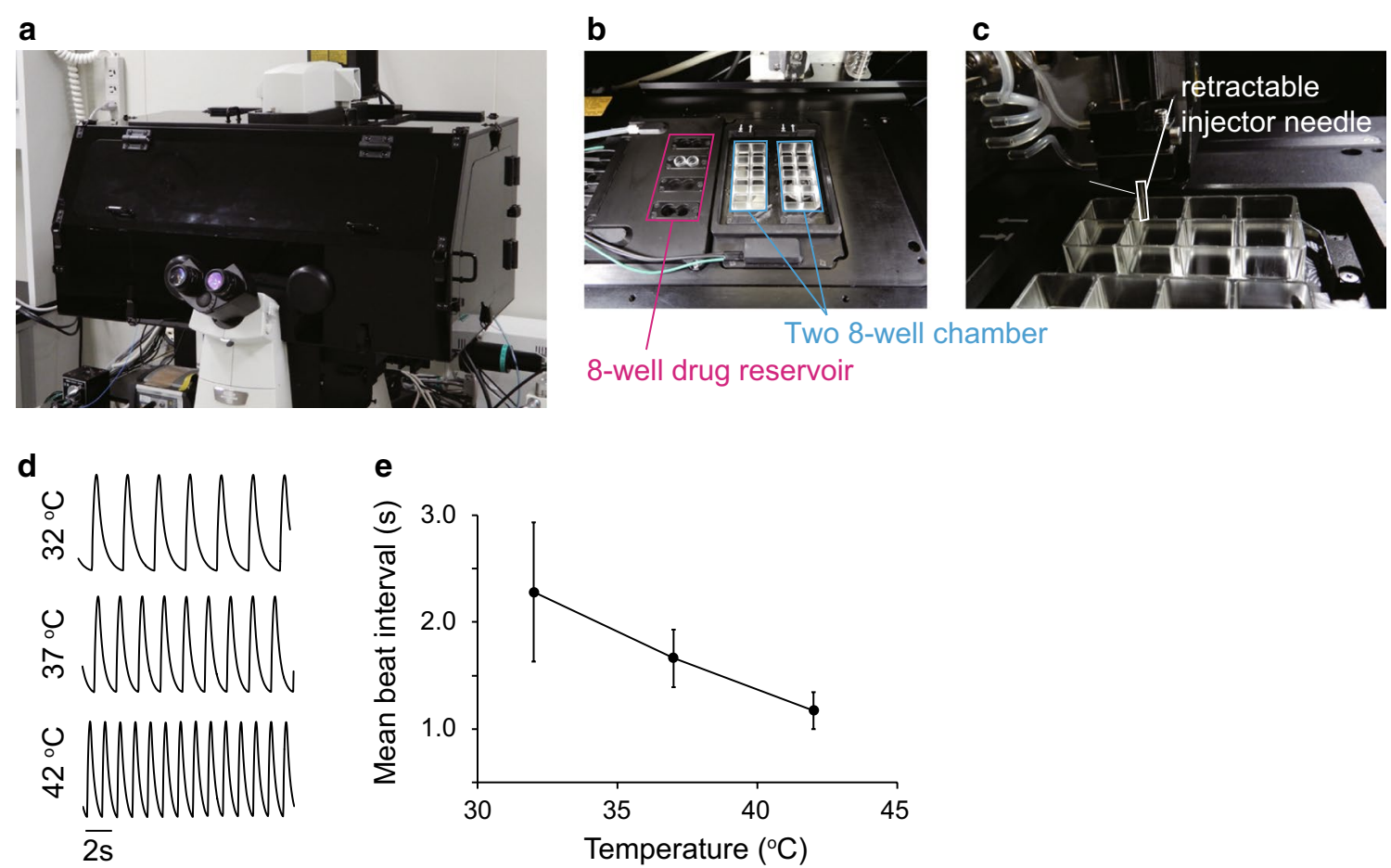

Fig. 2 Stage customisation for environmental control, and drug addition during bioluminescence imaging. a The observation area was covered with a light-tight box, b with stage adjustments to hold two 8-well chamber slides (cyan) and eight drug reservoirs (magenta), c the automatic dispenser system inside the light-tight box. $\mathbf{d}$ time course of Fluo-4 fluorescence intensity in hiPSC-CM at 32, 37 , and $42{ }^{\circ} \mathrm{C}$. The measurements were triplicated at each temperature, and representative data are shown. e Temperature dependency of peak-to-peak interval of hiPSC-CM. The peak-to-peak interval decreased as temperature was raised from 32 to $42^{\circ} \mathrm{C}$. Data points show the mean \pm s.e, $n=3$

incubator itself has two spaces to hold an 8 well chamber slide for cell observation and eight holes acting as drug reservoirs (Fig. 2c). Temperature can be controlled independently for cell observation $\left(25-50{ }^{\circ} \mathrm{C}\right)$ and drug storage $\left(4-50{ }^{\circ} \mathrm{C}\right)$. Since beating parameters of hiPSC-CMs exhibit thermal dependence, the temperature inside the chamber overall should be constant. To validate the performance of the incubator, $\mathrm{Ca}^{2+}$ transients of hiPSC-CMs were recorded by Fluo4, a commonly used fluorescent chemical indicator for $\mathrm{Ca}^{2+}$, at 32,37 and $42{ }^{\circ} \mathrm{C}$. Figure $2 \mathrm{~d}$ shows a typical time course of spontaneous $\mathrm{Ca}^{2+}$ transients at each temperature. The peak-to-peak interval between $\mathrm{Ca}^{2+}$ transients, commonly employed as a measure of beat rate, decreased linearly from $2.8 \pm 0.29 \mathrm{~s}$ at $32{ }^{\circ} \mathrm{C}$ to $1.2 \pm 0.30 \mathrm{~s}$ at $42{ }^{\circ} \mathrm{C}$ (Fig. $2 \mathrm{e}, \mathrm{n}=3$ ).

\section{hiPSC-CM imaging}

We then assessed Gamillus-based $\mathrm{NL}\left(\mathrm{Ca}^{2+}\right) \Delta 9$ [hereafter $\mathrm{GmNL}\left(\mathrm{Ca}^{2+}\right)$ ] signals during spontaneous hiPSC-CM contraction in the customized microscope. $\mathrm{GmNL}\left(\mathrm{Ca}^{2+}\right)$ was expressed in cardiomyocyte spheroids by Adeno associated virus infection (Fig. 3a). $\operatorname{GmNL}\left(\mathrm{Ca}^{2+}\right)$ was imaged at $20 \mathrm{~Hz}$ for $15 \mathrm{~min}$, detecting periodic changes in bioluminescence signal during synchronized contractions (Fig. 3b). The time course of bioluminescence intensity was recorded for the first and last $3 \mathrm{~min}$, and analyzed to estimate three beating parameters; peak interval, peak amplitude, and 50\% peak width (FWHM) which approximates to the action potential duration. We compared these results to those obtained using Fluo4 under various illumination power densities (62, 125, 250 and $500 \mathrm{~mW} \mathrm{~cm}^{-2}$ respectively). The amplitude of $\mathrm{GmNL}\left(\mathrm{Ca}^{2+}\right)$ did not change significantly during observation $(-8.0 \pm 21 \%, \mathrm{n}=8)$, in contrast to that of Fluo4 which reduced significantly under all illumination conditions tested $\left(-50 \pm 8.5 \%\right.$ at $62 \mathrm{~mW} \mathrm{~cm} \mathrm{~cm}^{-2}$ to $-99 \pm 0.51 \%$

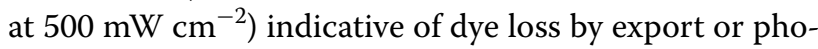
tobleaching (Fig. 3c).

In addition, stability of beating parameters between the start and the end of the observation window was only seen with $\mathrm{GmNL}\left(\mathrm{Ca}^{2+}\right.$ ) (From $1.3 \pm 0.36$ to $1.3 \pm 0.27 \mathrm{~s}$ for beat-beat interval, and $0.61 \pm 0.14$ to $0.59 \pm 0.063 \mathrm{~s}$ for FWHM, $\mathrm{n}=8$ ) and Fluo4 at the lowest power density $\left(62 \mathrm{~mW} \mathrm{~cm}^{-2}\right.$ ) (from $1.6 \pm 0.11$ to $1.8 \pm 0.10 \mathrm{~s}$ for inter$\mathrm{val}$, and from $0.48 \pm 0.029$ to $0.59 \pm 0.031 \mathrm{~s}$ for FWHM, $\mathrm{n}=6$ ). At higher power density reduction in peak-topeak interval (From $1.9 \pm 0.10$ to $0.99 \pm 0.051 \mathrm{~s}$ at 500 $\mathrm{mW} \mathrm{cm}^{-2}, \mathrm{n}=7$ ) was seen suggesting extrinsic light 

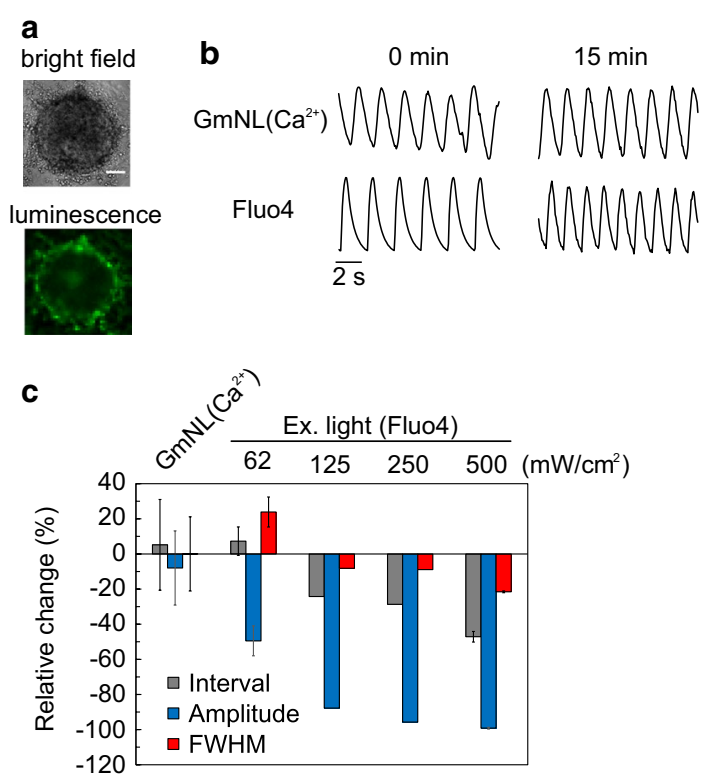
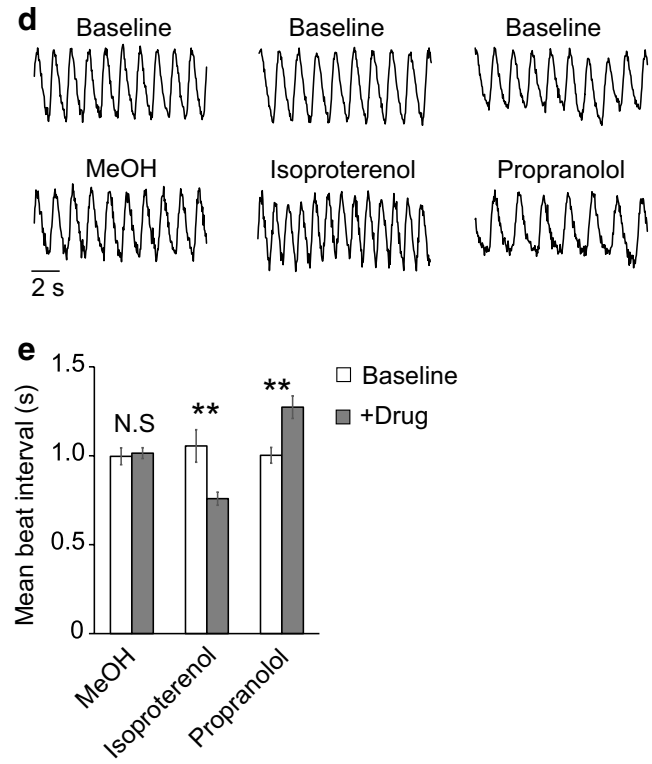

Fig. $3 \mathrm{Ca}^{2+}$ imaging with $\mathrm{GmNL}\left(\mathrm{Ca}^{2+}\right)$ in hiPSC-CMs. a Bioluminescence and bright-field images of hiPSC-CMs expressing $\mathrm{GmNL}\left(\mathrm{Ca}^{2+}\right)$. Scale bar, $50 \mu \mathrm{m}$. b Representative time course of the signal of $\mathrm{GmNL}\left(\mathrm{Ca}^{2+}\right)$, and Fluo4 at 0 or $15 \mathrm{~min}$. Images were taken at $20 \mathrm{~Hz}, 20 \mu \mathrm{M}$ coelenterazine-h was added just before imaging. Excitation light at $62 \mathrm{~mW} \mathrm{~cm}{ }^{-2}$ for Fluo4 was applied continuously for $15 \mathrm{~min}$. The measurements were replicated $(n \geq 6)$ for each condition. c Relative change in beating parameters after $15 \mathrm{~min}$ continuous imaging. Data are presented as mean \pm S.D.; $n=6-8$. $\mathrm{Ca}^{2+}$ imaging with Fluo4 was conducted under four different power densities of excitation light, 62, 125, 250 and $500 \mathrm{~mW} \mathrm{~cm}^{-2}$ respectively. $\mathbf{d}$ Representative time course of the bioluminescence signal of $\mathrm{GmNL}\left(\mathrm{Ca}^{2+}\right)$ before and after treatment with either $1 \mu \mathrm{M}$ isoproterenol or $40 \mu \mathrm{M}$ propranolol. The measurements were triplicated for each drug. e Mean beat interval before and after treatment; data are presented as mean \pm S.D. Two-tailed Student's t test was performed. ${ }^{* *} p<0.01 ; n=3$ illumination can cause significant phototoxicity in this system (Fig. 3c). Collectively, these observations demonstrate that $\mathrm{GmNL}\left(\mathrm{Ca}^{2+}\right)$ improves long-term signal stability without physiological perturbations arising from extrinsic illumination. Interestingly the variation between hiPSC-CMs from $\mathrm{GmNL}\left(\mathrm{Ca}^{2+}\right)$ was greater than that from cells labelled with Fluo4.

\section{Drug-induced changes to the hiPS-CM $\mathrm{Ca}^{2+}$ transient}

Next we tested whether $\mathrm{GmNL}\left(\mathrm{Ca}^{2+}\right)$ can identify expected $\mathrm{Ca}^{2+}$ transient changes in hiPSC-CMs induced by well characterized drugs. After addition of isoproterenol, a non-selective $\beta$-adrenergic agonist used clinically to increase the heart rate, the mean peak-to-peak interval reduced (from $1.1 \pm 0.16$ to $0.75 \pm 0.070 \mathrm{~s}, \mathrm{n}=3$ ) (Fig. 3d, e) [13]. Conversely, propranolol, an adrenergic receptor blocker, increased the peak-to-peak interval (from $1.0 \pm 0.081$ to $1.27 \pm 0.11 \mathrm{~s}, \mathrm{n}=3$ ) as expected (Fig. 3d, e) [14]. Similarly $\mathrm{GmNL}\left(\mathrm{Ca}^{2+}\right)$ could correctly elicit the rate related $\mathrm{Ca}^{2+}$ transient alterations induced by Dopamine, and Doxazosin (Additional file 4). These results suggested that $\mathrm{GmNL}\left(\mathrm{Ca}^{2+}\right)$ is able to report bidirectional drug effects in hiPSC-CMs using a drug dispensing system based inside the on-stage environmental control conditions needed for bioluminescence imaging.

\section{Discussion}

$\mathrm{GmNL}\left(\mathrm{Ca}^{2+}\right)$ enables imaging free from the problems of phototoxicity and photobleaching, which plague fluorescence imaging. In contrast to our expectations, $\mathrm{GmNL}\left(\mathrm{Ca}^{2+}\right)$ was slightly dimmer than Nanolantern $\left(\mathrm{Ca}^{2+}\right)$ at saturating $\mathrm{Ca}^{2+}$ concentrations. Saturation mutagenesis at the junction between the light donor and the FRET acceptor might improve the performance of $\mathrm{GmNL}\left(\mathrm{Ca}^{2+}\right)$. Although the $\mathrm{GmNL}\left(\mathrm{Ca}^{2+}\right)$ measurements in hiPSC-CM show stable $\mathrm{Ca}^{2+}$ transients between the beginning and the end of the observation window, the variation between hiPSC-CMs appears high in comparison with that from Fluo4. The observed variability might be attributable to heterogeneous infection of $\mathrm{AAV}$ or reduced penetration of the luminescent substrate into the spheroid culture model as either may lower bioluminescence signal.

The spontaneous beating characteristics of cardiomyocytes are sensitive to the physical environment, especially temperature, therefore maintenance of sample environment is crucial. In our system we can independently control the temperature of the sample chamber and the drug reservoir, remotely adding the small molecules without perturbation of imaging environment.

Overall, our study presents a bioluminescent $\mathrm{Ca}^{2+}$ indicator and a light-tight box equipped with an automatic dispenser that can be controlled remotely. As a 
proof-of-concept, we demonstrate a minimally harmful, and operator independent $\mathrm{Ca}^{2+}$ imaging strategy in hiPSC-CMs with robust testing of drug-induced changes in $\mathrm{Ca}^{2+}$ transients at a scale.

\section{Limitations}

As $\mathrm{GmNL}\left(\mathrm{Ca}^{2+}\right)$ is intensiometric indicator, the oscillation from $\mathrm{GmNL}\left(\mathrm{Ca}^{2+}\right)$ in hiPSC-CMs should include the fraction of motion artefact in addition to $\mathrm{Ca}^{2+}$-dependent signal as previously shown [7]. A negative control using $\mathrm{Ca}^{2+}$-insensitive probe will give the insightful information about motion artefact.

\section{Additional files}

Additional file 1. Oligonucleotides used in this study.

Additional file 2. Drugs used in this study.

Additional file 3. Characterization of the bioluminescent $\mathrm{Ca}^{2+}$ indicators in HeLa. (a) A series of pseudo-coloured ratio images of HeLa cells expressing $\mathrm{GmNL}\left(\mathrm{Ca}^{2+}\right)$, following $10 \mu \mathrm{M}$ histamine stimulation (arrow). Scale bar, $10 \mu \mathrm{m}$. (b) Time course of the $B / B_{0}$ ratio change at an $\mathrm{ROI}$ (white box in (a)). Number indicates the time point of each image in (a).

Additional file 4. Evaluation of mean beat interval before and after treatment with either $10 \mu \mathrm{M}$ Dopamine or $10 \mu \mathrm{M}$ Doxazosin. Two-tailed Student's t-test was performed. ${ }^{* *} p<0.01$; Data are presented as mean \pm S.D.; $n=3$.

\section{Abbreviations}

hiPSC: human-induced pluripotent stem cells; CM: cardiomyocytes; BP: bioluminescent protein; GFP: green fluorescent protein; AAV: adeno-associated virus; QY: quantum yield; FWHM: full width at half maximum; Rluc: Renilla luciferase; FRET: Förster resonance energy transfer.

\section{Authors' contributions}

$\mathrm{KS}$ and TO contributed equally to this work. TN conceived and coordinated the project; KS, TO, MN, TM, CN and ST designed the experiments; CN and ST optimized the microscopy; KS and TO constructed and characterized GNL(Ca ${ }^{2+}$ ) in vitro, and performed the $\mathrm{Ca}^{2+}$ imaging of hiPSC-CM with support of CN and ST; TO assayed the drug-induced alteration of $\mathrm{Ca}^{2+}$ transient in hiPSC-CM with support of CN, ST and MJD; all authors analyzed data; KS, TO, CN, TM and TN wrote the paper, with contributions from all authors. All authors read and approved the final manuscript.

\section{Author details}

${ }^{1}$ The Institute of Scientific and Industrial Research, Osaka University, 8-1 Mihogaoka, Ibaraki, Osaka 567-0047, Japan. ${ }^{2}$ NIKON CORPORATION, 471, Nagaodai-cho, Sakae-ku, Yokohama, Kanagawa 244-8533, Japan. ${ }^{3}$ BHF Centre for Regenerative Medicine, Division of Cardiovascular Medicine, West Wing Level 6, John Radcliffe Hospital, Oxford University, Oxford OX3 9DU, UK.

\section{Acknowledgements}

The authors wish to thank Dr. Yamada from Nikon cooperation for their help in preparation of hiPSC-CM. pAAV-CAG-ArchT-GFP (Addgene plasmid \# 29777) was a gift from Edward Boyden.

\section{Competing interests}

The authors declare that they have no competing interests.

\section{Availability of data and materials}

The data that support the findings of this study are available from the corresponding author on request. The nucleotide sequences of Gamillus-based $\mathrm{NL}\left(\mathrm{Ca}^{2+}\right)$ _variants have been deposited to DDBJ database under the following entry IDs: LC325493 (Gamillus-based NL(Ca2 +)__C8), LC325494 (Gamillus-based

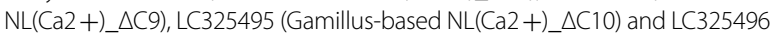
(Gamillus-based NL(Ca2+)_ $\triangle \mathrm{C} 11)$, respectively.

\section{Consent for publication}

Not applicable.

Ethics approval and consent to participate

Not applicable.

Funding

Not applicable.

\section{Publisher's Note}

Springer Nature remains neutral with regard to jurisdictional claims in published maps and institutional affiliations.

Received: 12 December 2017 Accepted: 10 May 2018

Published online: 18 May 2018

\section{References}

1. Takahashi K, Yamanaka S. Induction of pluripotent stem cells from mouse embryonic and adult fibroblast cultures by defined factors. Cell. 2006;126(4):663-76.

2. Takahashi K, Tanabe K, Ohnuki M, Narita M, Ichisaka T, Tomoda K, et al. Induction of pluripotent stem cells from adult human fibroblasts by defined factors. Cell. 2007;131(5):861-72.

3. Mercola M, Colas A, Willems E. Induced pluripotent stem cells in cardiovascular drug discovery. Circ Res. 2013;112(3):534-48.

4. Fermini B, Hancox JC, Abi-Gerges N, Bridgland-Taylor M, Chaudhary KW, Colatsky T, et al. A new perspective in the field of cardiac safety testing through the comprehensive in vitro proarrhythmia assay paradigm. J Biomol Screen. 2016;21(1):1-11.

5. Daily NJ, Santos R, Vecchi J, Kemanli P, Wakatsuki T. Calcium transient assays for compound screening with human iPSC-derived cardiomyocytes: evaluating new tools. J Evol Stem Cell Res. 2017;1(2):1-11.

6. Harris K, Aylott M, Cui Y, Louttit JB, McMahon NC, Sridhar A. Comparison of electrophysiological data from human-induced pluripotent stem cellderived cardiomyocytes to functional preclinical safety assays. Toxicol Sci. 2013:134(2):412-26.

7. Kaestner L, Scholz A, Tian Q, Ruppenthal S, Tabellion W, Wiesen K, et al. Genetically encoded $\mathrm{Ca}^{+}{ }^{+}$indicators in cardiac myocytes. Circ Res. 2014;114(10):1623-39

8. Shinnawi R, Huber I, Maizels L, Shaheen N, Gepstein A, Arbel G, et al. Monitoring human-induced pluripotent stem cell-derived cardiomyocytes with genetically encoded calcium and voltage fluorescent reporters. Stem Cell Rep. 2015;5(4):582-96

9. Saito K, Chang YF, Horikawa K, Hatsugai N, Higuchi Y, Hashida M, et al. Luminescent proteins for high-speed single-cell and whole-body imaging. Nat Commun. 2012:3:1262.

10. Takai A, Nakano M, Saito K, Haruno R, Watanabe TM, Ohyanagi T, et al. Expanded palette of nano-lanterns for real-time multicolor luminescence imaging. Proc Natl Acad Sci USA. 2015;112(14):4352-6.

11. Suzuki K, Kimura T, Shinoda H, Bai G, Daniels MJ, Arai Y, et al. Five colour variants of bright luminescent protein for real-time multicolour bioimaging. Nat Commun. 2016;7:13718.

12. Shinoda H, Ma Y, Nakashima R, Sakurai K, Matsuda T, Nagai T. Acid-tolerant monomeric GFP from Olindias formosa. Cell Chem Biol. 2018;25(3):330. e7-338.e7.

13. Mandel Y, Weissman A, Schick R, Barad L, Novak A, Meiry G, et al. Human embryonic and induced pluripotent stem cell-derived cardiomyocytes exhibit beat rate variability and power-law behavior. Circulation. 2012;125(7):883-93.

14. Heylman C, Datta R, Sobrino A, George S, Gratton E. Supervised machine learning for classification of the electrophysiological effects of chronotropic drugs on human induced pluripotent stem cell-derived cardiomyocytes. PLOS ONE. 2015;10(12):e0144572. 